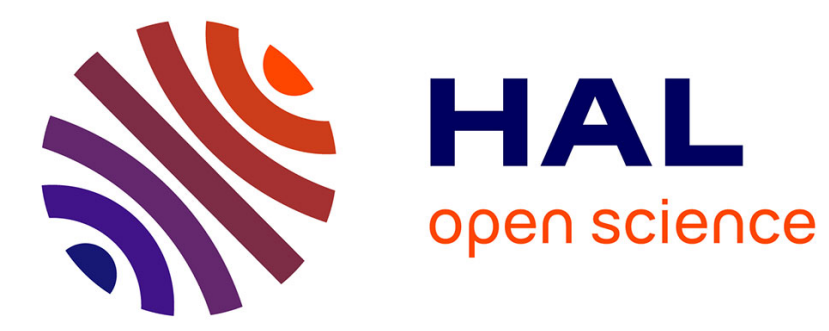

\title{
À propos des collectifs d'usagers de la santé en France au 20 ème siècle
}

Sandrine Knobé

\section{To cite this version:}

Sandrine Knobé. À propos des collectifs d'usagers de la santé en France au 20 ème siècle. Global Health Promotion, 2010, 17 (3), pp.85-88. 10.1177/1757975910375176 . hal-02542896

\section{HAL Id: hal-02542896 https://hal.science/hal-02542896}

Submitted on 14 Apr 2020

HAL is a multi-disciplinary open access archive for the deposit and dissemination of scientific research documents, whether they are published or not. The documents may come from teaching and research institutions in France or abroad, or from public or private research centers.
L'archive ouverte pluridisciplinaire HAL, est destinée au dépôt et à la diffusion de documents scientifiques de niveau recherche, publiés ou non, émanant des établissements d'enseignement et de recherche français ou étrangers, des laboratoires publics ou privés. 


\section{À propos des collectifs d'usagers de la santé en France au 20ème siècle}

\section{Pour citer cet article :}

Knobé S., «À propos des collectifs d'usagers de la santé en France au $20^{\text {ème }}$ siècle », Journal of global health promotion, vol.17, $\mathrm{n}^{\circ} 3,2010$, pp.85-88.

\section{Résumé :}

L'expression collective de doléances propres aux usagers de la santé n'émerge pas subitement dans les années 1980-1990 avec les actions associatives très médiatisées comme celles de la lutte contre le sida. Mais cette période s'avère toutefois propice à une politisation progressive de la question de la participation des usagers au processus d'élaboration des politiques publiques de santé du fait, entre autres, de la médiatisation de différentes affaires dites de santé publique. La thématique de la participation des usagers au système de santé prend ainsi place dans le débat politique et connaît plusieurs mises en forme juridique jusqu'à devenir un enjeu reconnu sous l'appellation de «démocratie sanitaire ». Cet article se propose de donner des points de repères quant à l'histoire en France des collectifs porte-parole des usagers de la santé des années 1970 à nos jours.

Mots clés : France, représentation, usagers 
En France, pour l'élaboration de la loi «hôpital, patients, santé et territoires » (HPST), les pouvoirs publics ont décidé d'engager divers cycles d'échanges avec, et entre, une grande diversité d'acteurs : professionnels de la santé libéraux et hospitaliers, représentants du secteur médicosocial, élus, représentants des usagers, experts... Ces concertations se sont déroulées dans le cadre de la préparation de quatre rapports demandés par la ministre de la Santé, ainsi qu'à l'occasion des états généraux de l'organisation de la santé. La volonté affichée de permettre aux usagers de s'exprimer et de participer au processus d'élaboration des politiques publiques de santé paraît ici aller de soi. Pourtant, un tel état de fait n'a été rendu possible que par un travail militant associatif dont la légitimité n'est reconnue que tardivement au cours des années 1990. Nous nous proposons ici de rappeler les différentes étapes qui jalonnent cette reconnaissance.

L'histoire, en France, du mouvement associatif dans le domaine de la santé connaît trois grandes périodes (1). La première, qui correspond au $19^{\text {ème }}$ siècle, est celle de la charité à l'égard de certaines catégories d'indigents malades ne trouvant leur place ni à l'hôpital ni à l'hospice. La deuxième période, qui couvre la fin du $19^{\text {ème }}$ et le début du $20^{\text {ème }}$ siècle, inscrit la lutte contre la maladie dans celle contre les fléaux sociaux, et voit ainsi apparaître les «ligues » contre la tuberculose, la syphilis, l'alcoolisme ou le cancer. Le ministère de l'Intérieur engage des actions en termes d'hygiène publique tendant, notamment par l'intermédiaire des bureaux d'hygiène municipaux, à la mise en place d'une surveillance épidémiologique et à la diffusion, à l'intention des couches populaires, des bonnes manières hygiéniques (2). La France est très active pendant cette période dans ce domaine de l'hygiène (3) et émerge, comme dans d'autres pays européens (dont l'Angleterre et l'Allemagne), l'idée de «santé publique » selon laquelle la mise en œuvre de politiques sociales et sanitaires permettrait une meilleure gestion de la santé tant au niveau public, que privé (4). Enfin, la troisième période, qui démarre après la seconde guerre mondiale, se caractérise par une plus grande diversité des logiques d'action : collecte de 
fonds destinés à la recherche biomédicale, gestion de structures de prise en charge, et autoorganisation des usagers sur le modèle des groupes de self-help développés aux États-Unis. Apparaissent ainsi des associations aux objectifs plus diversifiés et ne fonctionnant pas nécessairement ou uniquement selon une logique philanthropique. Dans le domaine du handicap physique et mental, des associations particulièrement actives mettent en place et gèrent des structures de prise en charge spécialisées, et contribuent également à la reconnaissance de droits spécifiques aux personnes handicapées. Il n'est alors pas question de la défense des droits des malades ou des usagers de la santé au sens large. La multiplication des associations dites d'entraide (5) qui caractérise les années 1970 s'inscrit également dans une tendance à l'organisation sectorielle par pathologie. Ces associations sont créées par des patients eux-mêmes, et fonctionnent selon une logique d'entraide entre anciens et nouveaux patients. L'accent est alors mis sur le partage d'une expérience commune en mesure d'apporter soutien moral et matériel. Par ailleurs, à l'initiative de femmes décidées à développer une gestion autonome de leur santé, se développent des groupes plus contestataires. Ces associations s'éloignent du modèle strict de l'entraide mutuelle pour revendiquer le droit à l'information et au libre-choix des thérapeutiques, notamment celles dites parallèles. Elles défendent l'idée d'un malade autosoignant (6) pour qui la prise en charge autonome de la maladie va jusqu'à l'acquisition d'un savoir scientifique et technique rivalisant avec celui du médecin. L'enjeu est alors essentiellement individuel, et consiste dans la gestion au quotidien d'une pathologie chronique. La question d'une participation des usagers au processus d'élaboration des politiques publiques de santé n'est pas encore d'actualité.

On aurait cependant pu penser en trouver les prémisses dans la mise en place dès 1974, sous la présidence de Valéry Giscard d'Estaing, d'un Comité d'usagers du Ministère de la Santé (7). Mais cette action gouvernementale a été éphémère, n'a eu qu'un impact limité par rapport au secteur hospitalier, et participait davantage d'une logique consultative que représentative. Si ce 
Comité ne fait pas mention ou ne préconise pas une participation directe des usagers aux décisions concernant le fonctionnement de l'institution hospitalière, un rapport de l'Inspection Générale des Affaires Sociales (8) quelques années plus tard la recommande fortement dans l'intention de prévenir d'éventuels contentieux entre les usagers et l'institution hospitalière. Cette incitation à la participation ne reste toutefois qu'à l'état de projet, et devient alors le leitmotiv des revendications portées par les collectifs d'usagers à vocation représentative qui se constituent au cours des années 1980. À titre d'exemple, on peut citer la constitution en 1976 d'un Ordre national des patients, la création en 1981 d'une Fédération nationale des groupes d'usagers de la santé, la formation en 1987 de Réshus (réseau hospitalier des usagers pour la représentation des associations d'usagers dans l'hôpital), en 1989 de AUHSM-Santé défense (association des usagers de l'hôpital et des soins médicaux) ou encore en 1992 du GAUM (groupe d'associations d'usagers de la médecine). Ces collectifs ne réussissent cependant pas à s'instituer porte-parole des usagers de la santé tant dans l'univers associatif que dans leurs relations avec les instances politiques et administratives. Très confidentiels et souvent cantonnés à un secteur géographique restreint, ils ne parviennent pas à trouver suffisamment de relais médiatiques ou politiques susceptibles d'accroître l'audience et la portée de leurs revendications. Tout à l'inverse de ce que vont accomplir les associations de lutte contre le sida et notamment les deux plus importantes en France : Aides (créée en 1984) et Act-Up (créée en 1989). Cette dernière se démarque notamment par des modes d'actions militants plus virulents et provocateurs.

C'est dans ce contexte des années sida qu'éclatent également plusieurs affaires dites de santé publique, qui vont offrir un terreau propice à la politisation de la question de la participation des usagers au processus d'élaboration des politiques publiques de santé. Le drame du sang contaminé, qui en représente l'exemple le plus significatif, a fortement marqué l'opinion publique ainsi que les acteurs politiques eux-mêmes, à travers la mise en cause de 
responsabilités au plus haut niveau de l'État. Tout au long des années 1990, d'autres affaires vont tour à tour connaître une médiatisation importante, portées ou non par des collectifs porteparole de victimes, parmi lesquelles les affaires relatives à l'hormone de croissance (1992), à l'amiante (1995), à la vache folle (1996) ou au retraitement des déchets radioactifs des installations nucléaires de La Hague (1997). La dramaturgie de ces affaires, où les témoignages de victimes occupent une place de plus en plus importante, contribue à créer des conditions favorables à la naissance et à la réussite de mouvements contestataires portés par des usagers eux-mêmes ou leurs représentants. En réaction à ces scandales sanitaires, des rapports d'informations sont rapidement commandités par les pouvoirs publics en vue de désamorcer les crises de santé publique susceptibles de succéder à ces affaires très médiatisées. Les médias mettent dans ce contexte l'accent sur des témoignages individuels renforçant la dramaturgie de ces affaires (9). Dans le cadre de telles enquêtes publiques, les associations de victimes ou représentant les usagers sont en général auditionnées afin d'exposer leurs principales revendications.

Les années 1990 sont également synonymes de changements politiques et de projets réformateurs dans le secteur de la santé. En 1995, à l'initiative d'Alain Juppé, Premier Ministre, est ainsi mis en place un Haut conseil de la réforme hospitalière chargé d'énoncer des propositions en ce domaine. Il préconise alors le renforcement des droits et de la participation des usagers des structures d'hospitalisation aux prises de décision (10); ce qui se traduit notamment par la nomination de représentants des usagers au sein des conseils d'administrations des hôpitaux publics. Une quinzaine d'associations (11) ayant été auditionnées dans le cadre de cette réflexion sur la réforme hospitalière décident alors de constituer un Collectif interassociatif sur la santé (CISS), avec pour ambition de suivre l'application des nouvelles mesures, et de constituer une force d'observation critique et de proposition en matière sanitaire et sociale. En prenant appui sur l'idée que, laissés aux seules 
mains d'experts scientifiques et politiques, les systèmes de prise de décision et de contrôle sanitaires ont montré leurs failles, le CISS revendique une participation réelle et accrue des usagers, premiers concernés. Conscients des bénéfices qu'ils pourraient tirer d'une collaboration avec le CISS, les pouvoirs publics invitent d'ailleurs ses représentants, en mai 1999, à un colloque au Sénat intitulé « Le pouvoir des malades » (12).

La notoriété des organisations d'envergure nationale qui constituent le CISS lui permet d'emblée d'apparaître aux yeux des pouvoirs publics comme un interlocuteur légitime et crédible. Affichant publiquement sa vocation de transversalité, le CISS cherche spécifiquement à défendre les droits des usagers du système de santé et à accroître leur participation aux prises de décision. Ses revendications vont être entendues au niveau politique, comme en atteste la publication d'un certain nombre de rapports d'expertise sur la question. Ainsi en est-il par exemple du rapport Evin sur les droits de la personne malade (1996), du rapport du comité national d'orientation des états généraux de la santé (1999) et du rapport Caniard sur la place des usagers dans le système de santé (2000). L'ensemble de ces rapports d'expertise envisage les questions de santé et en particulier celle de la participation des usagers dans une perspective globale et non pas spécifiquement centrée sur une pathologie donnée. La dimension politique de la participation des usagers, ainsi que l'ensemble du travail militant du CISS sont alors consacrés par la mise en forme juridique de cette question avec la promulgation de la loi du 4 mars 2002 relative «aux droits des malades et à la qualité du système de soin ». Cette loi renforce les droits individuels du malade dans le cadre de sa prise en charge (information, consentement, accès au dossier médical personnel...), et réaffirme la nécessaire participation des usagers en tant qu' acteurs institutionnels ayant un rôle spécifique à tenir, au sein notamment des commissions des relations avec les usagers et de la qualité de la prise en charge. Formulée et défendue par Bernard Kouchner, alors Ministre délégué chargé de la Santé, l'idée de démocratie sanitaire préconise la participation de tous les acteurs concernés, y compris et 
surtout les usagers eux-mêmes, aux décisions concernant le système de santé. L'inscription de cette problématique à l'agenda politique correspond à un tournant majeur, après la seconde guerre mondiale, dans l'histoire des collectifs d'usagers de la santé en France.

Grâce à une stratégie d'union entre associations mettant en avant la transversalité des questions touchant aux droits des malades quelles que soient les pathologies, les revendications de démocratie sanitaire ont trouvé une issue favorable. La représentation des usagers construite de la sorte par le CISS a été reconnue légitime dans la mesure où la question générale des droits des malades était présentée comme concernant actuellement ou potentiellement l'ensemble des citoyens et n'était pas restreinte à l'une ou l'autre pathologie exclusivement. Elle a ainsi été présentée comme détachée d'intérêts spécifiques (13). S'il reste des obstacles (14) à une participation effective des usagers au système de santé - notamment l'asymétrie de la relation médecin-malade, le flou de la définition même de l'usager, la construction historique du système de prise en charge comme étant l'affaire des médecins et la mixité des types de structures de santé, à la fois publiques et privées - son principe est maintenant inscrit dans la loi. Un palier supplémentaire a d'ailleurs été franchi en 2005 avec la mise en place d'une commission nationale d'agrément des associations représentant les usagers dans les instances hospitalières ou de santé publique. Le défi des années à venir consiste pour les représentants des usagers à dépasser le statut d'alibi de l'institution qui leur est encore souvent conféré. Le CISS développe actuellement à cet effet toute une offre de formation à la représentation afin de fournir les outils juridiques et technico-administratifs indispensables à la compréhension et à la participation aux débats d'expertise auxquels sont conviés les représentants des usagers. 


\section{Références}

1. PINELL P, Pour une étude du mouvement associatif. Éléments d'analyse sociologique. Gestions Hospitalières. 1997; n³68: 498-504.

2. BOURDELAIS P, Les hygiénistes, enjeux, modèles et pratiques. Paris: Belin, 2001.

3. ACKERKNECHT E, Hygiene in France: 1815-1848. Bulletin of the history of medicine, $1948, \mathrm{n}^{\circ} 2: 117-155$.

4. FASSIN D, L’espace politique de la santé. Essai de généalogie. Paris: PUF, 1996.

5. ROMEDER J-M, Les groupes d'entraide et la santé. Nouvelles solidarités, Ottawa/Montréal: Conseil canadien de Développement social; 1989.

6. HERZLICH C, PIERRET J, Malades d'hier, malades d'aujourd'hui. Paris: Payot; 1984.

7. Arrêté du 19 décembre 1974 Du comité d'usagers placé auprès du ministre de la santé publique, JO du 20/12/1974, p.12823.

8. Inspection générale des affaires sociales, Les institutions sociales et les usagers. Paris: Ministère des Affaires sociales; 1982; 354.

9. ROMEYER H, La santé à la télévision : émergence d'une question sociale. Questions de communication. $2007, \mathrm{n}^{\circ} 11: 51-70$.

10. Rapport du Haut conseil de la réforme hospitalière. Paris: La Documentation Française; 1995.

11. AIDES ; Association des Paralysés de France (APF) ; Association Française contre les Myopathies (AFM) ; Association Française des Diabétiques (AFD) ; Association Française des Hémophiles (AFH); Association Française des Polyarthritiques (AFP); Confédération Syndicale des Familles (CSF) ; Familles Rurales ; Ligue Nationale Contre le Cancer (LNCC) ; Organisation Générale des Consommateurs (ORGECO) ; Union Féminine Civique et Sociale (UFCS) ; Union Nationale des Associations de Parents et Amis de Personnes Handicapées (UNAPEI) ; Union Nationale des Associations Familiales (UNAF) ; Vaincre la Mucoviscidose (VLM) ; Réshus.

12. PETTY F, BERGOGNE A, Le pouvoir des patients. Impact Médecin. 1999; 451: 6-16.

13. CONTANDRIOPOULOS D, A sociological perspective on public participation in health care. Social science \& medicine. 2004, n58: 321-330.

14. STEFFEN M, La santé : les bénéficiaires en dehors des réformes, in P. WARIN (dir), Quelle modernisation des services publics? Les usagers au cœur des réformes, Paris, La Découverte, 1997, 201-220. 